\title{
Efficacy and Tolerability of an Herbal Formulation for Weight Management
}

\author{
Judith S. Stern, ${ }^{1,2}$ Jan Peerson ${ }^{1,3}$ Artatrana T. Mishra, ${ }^{4}$ Venkata Sadasiva Rao Mathukumalli, ${ }^{5}$ \\ and Poorna Rajeswari Konda ${ }^{6}$ \\ ${ }^{3}$ Program in International and Community Nutrition, ${ }^{1}$ Department of Nutrition; Department of ${ }^{2}$ Internal Medicine; \\ University of California-Davis, Davis, California, USA. \\ ${ }^{4}$ Department of General Medicine, Nagarjuna Hospitals, Vijayawada, India. \\ ${ }^{5}$ Department of Internal Medicine, Alluri Sitarama Raju Academy of Medical Sciences (ASRAM), Eluru, India. \\ ${ }^{6}$ Suraksha Health Village, Vijayawada, India.
}

\begin{abstract}
The clinical effects and tolerability of a novel herbal formulation comprising the extracts of Sphaeranthus indicus and Garcinia mangostana were assessed in two similarly designed randomized, double-blind, placebo-controlled, clinical trials in 100 human subjects with a body mass index (BMI) between 30 and $40 \mathrm{~kg} / \mathrm{m}^{2}$. Participants were randomized into two groups receiving either $400 \mathrm{mg}$ of herbal blend twice daily or two identical placebo capsules. All subjects received three meals ( $2000 \mathrm{kcal} / \mathrm{day}$ ) throughout the study and walked 5 days a week for $30 \mathrm{~min}$. The primary outcome was reduction in body weight. Secondary outcomes were reduction in BMI and in waist and hip circumference. Serum glycemic, lipid, and adiponectin levels were also measured. Ninety-five subjects completed the trials, and data from these two studies were pooled and analyzed. At study conclusion (8 weeks), statistically significant reductions in body weight $(5.2 \mathrm{~kg} ; P<.0001)$, BMI $\left(2.2 \mathrm{~kg} / \mathrm{m}^{2} ; P<.0001\right)$, as well as waist $(11.9 \mathrm{~cm} ; P<.0001)$ and hip circumferences $(6.3 \mathrm{~cm} ; P=.0001)$ were observed in the herbal group compared with placebo. An increase in serum adiponectin concentration was also found in the herbal group versus placebo $(P=.0008)$ at study conclusion along with reductions in fasting blood glucose $(12.2 \%, P=.01)$, cholesterol $(13.8 \%, P=.002)$, and triglyceride $(41.6 \%, P<.0001)$ concentrations. No changes were seen across organ function panels, multiple vital signs, and no major adverse events were reported. The minor adverse events were equally distributed between the two groups. Our findings suggest that the herbal blend appears to be a well-tolerated and effective ingredient for weight management.
\end{abstract}

KEY WORDS: • adiponectin • clinical study • Garcinia mangostana • Sphaeranthus indicus • weight loss

\section{INTRODUCTION}

$\mathbf{O}$ BESITY ARISES FROM AN IMBALANCE in energy consumption over energy expenditure. ${ }^{1}$ At the cellular level, this imbalance results in an increase in the size and number of mature adipocytes. ${ }^{2-4}$ Current treatments for obesity include pharmacotherapy, bariatric surgery, and lifestyle modification. ${ }^{5}$ Pharmacotherapy involves the use of anti-obesity drugs such as phentermine and orlistat. ${ }^{6,7}$ While each intervention has its distinct advantages, it may also have significant adverse effects and limitations. ${ }^{8}$ For example, phentermine causes insomnia, increases blood pressure and pulse rate, while orlistat decreases absorption of fat soluble vitamins and causes abdominal pain, flatulence, and diarrhea. ${ }^{8}$ Although bariatric surgery may achieve sustained weight loss, its accessibility is limited to patients with a body

Manuscript received 11 July 2012. Revision accepted 19 February 2013

Address correspondence to: Judith S. Stern, ScD, Department of Nutrition, University of California Davis, 3150B Meyer Hall, Davis, CA 95616, USA, E-mail: jsstern@ ucdavis.edu mass index (BMI) of $\geq 40$ or patients with a BMI of $\geq 35$ who also experience severe obesity-related comorbidities. ${ }^{9}$ Due to these limited medical options and safety concerns, many people are gravitating to remedies having a better safety profile to achieve and to maintain reductions in body weight.

A plethora of diets have been proposed to treat obesity. Although some dietary approaches may result in short-term weight loss, most diets lack long-term compliance, leading to weight regain. Incorporating nonprescriptional antiobesity dietary supplements into lifestyle modification may facilitate both short-term and long-term weight loss. ${ }^{10}$ In 2005, American consumers spent more than 1.6 billion dollars on weight-loss supplements. ${ }^{11}$ Unfortunately, many of these weight management supplements are under scrutiny, because they lack the scientific evidence to support their claims.

With the goal of developing a potent but safe weight-loss ingredient of herbal origin, we screened a library of extracts from various medicinal plants for their anti-adipogenic and lipolytic activities. The adipogenic screening was evaluated 
by the in vitro differentiation of mouse 3T3-L1 preadipocytes to mature adipocytes. The lipolysis screening was performed with mature 3T3-L1 cells by monitoring the release of the lipolytic by-product, glycerol, into the cell culture medium. A blend composing extracts of the flower heads from Sphaeranthus indicus and the fruit rinds of Garcinia mangostana was shown to be effective in inhibiting adipogenesis while promoting lipolysis (unpublished data).

Here, we report the results of a pooled analysis combining data from two randomized, double-blind, placebo-controlled clinical studies that are designed to evaluate the efficacy and tolerability of the herbal blend mentioned earlier for managing body weight in obese human subjects with weight loss as the primary outcome.

\section{MATERIALS AND METHODS}

\section{Study material}

The herbal blend consists of aqueous alcohol extracts of the flower heads from $S$. indicus and the fruit rinds of G. mangostana. These individual extracts were concentrated and mixed together in a 3:1 ratio of $S$. indicus extract to G. mangostana extract to achieve a final product that contains 3\% 7-hydroxyfrullanolide and 2\% $\alpha$-mangostin. These two phytochemical markers were selected based on bioassay guided testing and serve as endogenous reference standards for controlling batch-to-batch consistency. All batches were tested for bioactivity as an additional quality control safeguard.

The herbal blend was analyzed with an ACQUITY UPLC H-Class (Waters Corp., Milford, MA, USA) reversed phase ultra performance liquid chromatography (UPLC) with a Phenomenex Kinetex $1.7 \mu \mathrm{m}, 100 \mathrm{~mm} \times 2.1 \mathrm{~mm} \mathrm{C} 18$ column (Torrence, CA, USA) maintained at $40^{\circ} \mathrm{C}$. An aliquot of the reference material and samples were prepared in acetonitrile. Linearity was demonstrated by a three-point calibration of the reference material. Samples were prepared at a concentration according to the reference material calibration levels. The sample solution was then sonicated and filtered through polytetrafluoroethylene filter into an autosampler vial. The samples were analyzed under a gradient elution with a linear gradient from $30 \%$ to $70 \%$ acetonitrile in $0.1 \%$ phosphoric acid for $7 \mathrm{~min}$ at a flow rate of $0.6 \mathrm{~mL} / \mathrm{min}$ and at $210 \mathrm{~nm}$. The column was equilibrated for an additional 2.5 min before each run (Fig. 1).

The herbal blend $(400 \mathrm{mg})$ was encapsulated in size zero hard gelatin capsules with excipients. Placebo capsules contained only excipients, and were identical in appearance, size, weight, and color to the active capsules. The herbal blend was provided by InterHealth Nutraceuticals, Inc. (Benicia, CA, USA) under a license agreement with Laila Nutraceuticals, (Vijayawada, India).

\section{Recruitment of subjects}

The two clinical trials were performed at Alluri Sitarama Raju Academy of Medical Sciences (ASRAM), Eluru, Andhra Pradesh, India from November 2009 to April 2010 (clinical trial registration number: ISRCTN45078827) and from March 2010 to July 2010 (clinical trial registration number: ISRCTN52261953). The study protocols were evaluated and approved by the ASRAM Institutional Review Board (IRB).

A summary of the clinical study design is provided in Figure 2. A total of 182 subjects participated in the selection process held at the Internal Medicine Department of the ASRAM Hospital by a questionnaire-based screening procedure. Out of the 182 candidates screened, 100 subjects who met the eligibility criteria were enrolled in the study (Table 1). Each participant voluntarily signed the IRB approved informed consent form. After recruitment, the subjects were randomly assigned to either the placebo or the herbal blend group.

\section{Study design}

The purpose of these two randomized, double-blind, placebo-controlled clinical studies was to evaluate the weightreducing efficacy of the herbal blend in obese subjects. The primary study outcome was reduction in body weight.

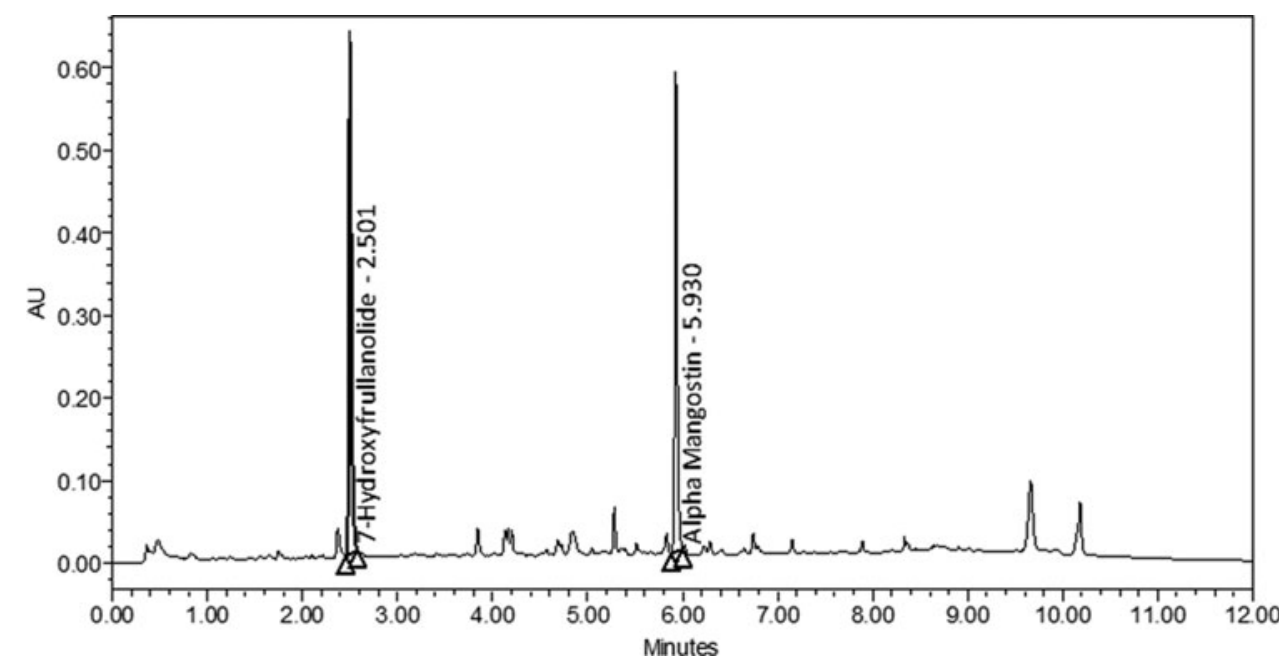

FIG. 1. Typical chromatogram of major components in the herbal blend as identified by standard ultra performance liquid chromatography (UPLC) procedures. The herbal blend was analyzed by a reverse-phase UPLC with a $\mathrm{C} 18$ column maintained at $40^{\circ} \mathrm{C}$. The samples were dissolved in acetonitrile. The main components, 7hydroxyfrullanolide and $\alpha$-mangostin were identified by standard UPLC procedures and detected at $210 \mathrm{~nm}$. The results were plotted in arbitrary units (AU) versus elution time. 


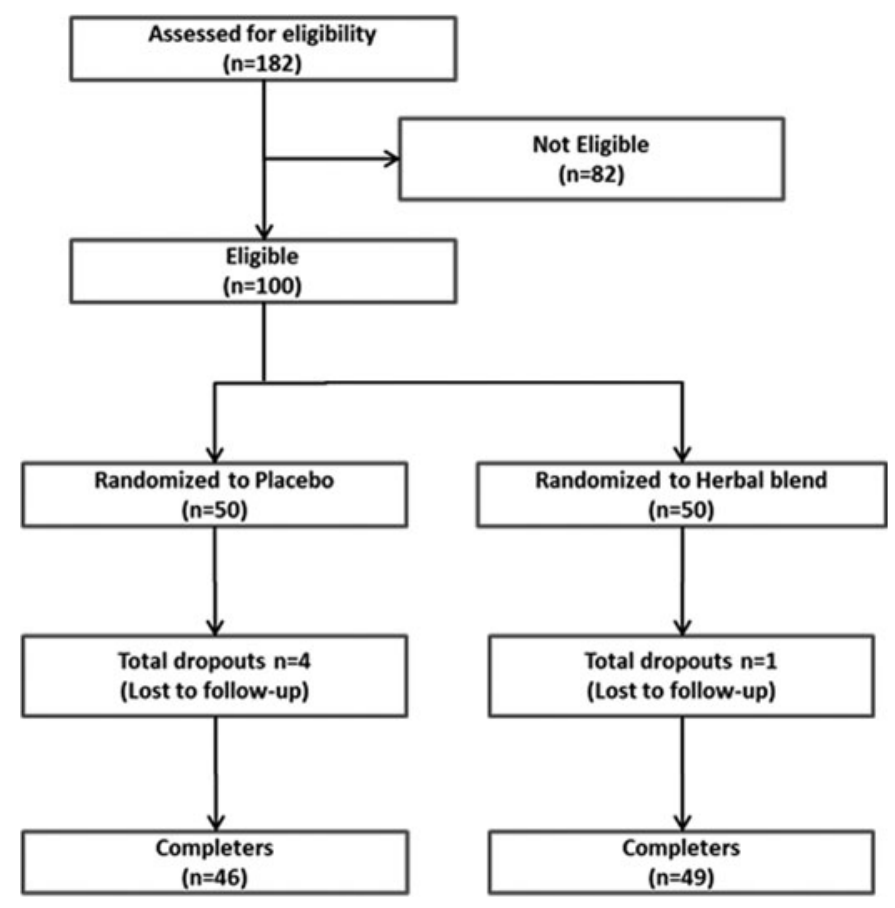

FIG. 2. Enrollment, randomization, and completion flow diagram.

At baseline evaluation, all the subjects were provided active or placebo capsules, compliance cards, the list of instructions for moderate exercise, schedules for delivery of daily meals, and dates for follow-up evaluations. The free prepared meals $(2000 \mathrm{kcal} / \mathrm{day})$ were provided in three portions: breakfast, lunch, and dinner. The daily caloric intake was as follows: $61 \%$ from carbohydrate, $14 \%$ from protein, and $25 \%$ from fat. The subjects were instructed to take two capsules a day, one each 30 min before breakfast and dinner.

All subjects completed a questionnaire regarding medical history and medication use, compliance with the exercise regimen, the diet at baseline, as well as all follow-up evaluations at 14, 28, and 56 days. In addition, all subjects completed a 27 -item version of the impact of weight on quality of life (IWQOL) questionnaire on Day 0 and 56. At baseline and subsequent follow-up visits, subjects were assessed for body weight, height, waist plus hip circumference, and vital signs. Blood and urine samples were collected at all evaluations and analyzed as described next.

\section{Hematological and biochemical evaluations}

The metabolic and lipid profile were evaluated in serum, while a complete blood count was done in whole blood at each visit. Serum biochemical parameters and hematological parameters were measured using the automated analyzer HumaStar 300 (HUMAN Diagnostics, Wiesbaden, Germany) and the hematological counter Humacount (HUMAN Diagnostics), respectively. The urine analysis was carried out using UroColor ${ }^{\mathrm{TM}} 10$ Dip Sticks and Urometer-600 (Standard Diagnostics, Kyonggi-do, Korea) and by microscopy of sediment.
Table 1. InClusion and ExClusion CRiteria

\section{Inclusion criteria}

Subjects should understand the risks and benefits of the protocol

Adults aged 21-50 years

BMI between 30 and $40 \mathrm{~kg} / \mathrm{m}^{2}$

Willingness to participate in the exercise-walking program, supervised by a trained exercise specialist

Willingness to consume the prescribed study diet of $\sim 2000 \mathrm{kcal} / \mathrm{day}$ as outlined in the protocol (meals provided free of cost by the study sponsor)

Written informed consent to participate in the trial

Willingness to complete standard health history questionnaire before recruitment into the study

Willingness to participate in five clinical visits (screening, baseline, 2, 4 and 8 weeks)

Subjects should abstain from alcoholic products during the study

If female: should be negative in pregnancy test if of child-bearing potential, and agree to follow an accepted method of birth control for the duration of the study such as condoms, foams, jellies, diaphragm, intrauterine device, etc.

\section{Exclusion criteria}

History of thyroid disease, cardiovascular disease, or diabetes

History of allergy to spices and herbal products

Body weight with $\mathrm{BMI}>40$

Nonobese $(\mathrm{BMI}<30)$

Using other weight loss medications, as well as stimulants, laxatives, or diuretics taken solely for the purpose of weight loss

Recent unexplained weight loss or gain

Positive HIV test

Undergone surgery before 30 days of screening or planning to undergo surgery within the study period

History of hepatitis, pancreatitis, lactic acidosis, or hepatomegaly with steatosis

History of motor weakness or peripheral sensory neuropathy

Any evidence of organ dysfunction or any clinically significant deviation from normal that could impact subjects' well-being or clinical outcome

BMI, body mass index.

\section{Cardiac assessments}

Cardiac functions were evaluated with Mortara ELI 250 (Milwaukee, WI, USA) and Schiller AT-2 instruments (Doral, FL, USA) according to instructions supplied by the manufacturers of the instruments and hospital protocols. Results were interpreted by hospital cardiologists.

\section{Serum adipokine assay}

Serum adiponectin levels were determined with human adiponectin enzyme-linked immunosorbent assay (ELISA) kit according to the procedure provided by the manufacturer (Millipore Corp., Billerica, MA, USA).

\section{Assessing the IWQOL}

The IWQOL is a self-report questionnaire that is specifically developed to assess the effect of obesity on quality of life. ${ }^{12}$ In the current study, an abridged version of the IWQOL questionnaire was used to assess the effect of herbal supplementation on the four scales of the IWQOL-Lite. ${ }^{13}$ The 27-item instrument consists of physical function (11 
items), self-esteem (7-items), public distress (5 items), and work (4 items) as previously described. ${ }^{13}$

\section{Statistical analysis}

Outcome variables were assessed for conformance to the normal distribution and transformed if necessary. Mean values of the outcomes were compared with a mixed model analysis, including treatment group and time as main effects, the interaction between treatment group and time, and baseline value of the variable and gender as covariates; follow-up analyses of covariance were used to compare the groups at each individual time point. For the Day 0 comparison, a $t$-test was used. $P$ values $<.05$ were considered statistically significant for between-group comparisons. All comparisons reported here are between the placebo and the herbal blend groups at each specified time point. Analysis was performed with SAS for Windows Release 9.2 (Cary, NC, USA). All statistical results were independently generated by the Department of Nutrition, University of California, Davis. For reference, normal ranges for the measured parameters were obtained from Medline and the Mayo Clinic. ${ }^{14,15}$

\section{RESULTS}

Study material fingerprinting and baseline demographic characteristics

The herbal blend was analyzed by HPLC, and the major peaks were identified in Figure 1. 7-hydroxyfrullanolide was eluted at $2.5 \mathrm{~min}$, and $\alpha$-mangostin was eluted at $5.9 \mathrm{~min}$.

Demographic and baseline characteristics of the study subjects are summarized in Table 2. The subjects' profiles with regard to age, sex, height, weight, BMI, waist circumference, hip circumference, blood pressure, and pulse rate were similar between the herbal blend $(800 \mathrm{mg} /$ day, $n=49)$ and placebo $(n=46)$ groups.

\section{Reduction in body weight and BMI}

Figure 3A summarizes body weight changes over time for subjects supplemented with the herbal blend or placebo. The herbal blend group experienced a statistically significant greater reduction in their body weight at 2,4 , and 8 weeks of supplementation compared with subjects consuming placebo $(P<.0001)$. The net reduction in body weight for both cohorts is summarized in Table 3 . The herbal blend group experienced a statistically significant net weight loss of 1.3 , 2.3 , and $3.8 \mathrm{~kg}$ at 2,4 , and 8 weeks, respectively. These net changes represent a $1.6 \%, 2.8 \%$, and $4.6 \%$ decrease in overall body weight. A similar decrease in BMI was observed for subjects consuming the herbal blend (Fig. 3B and Table 3). Supplementation with the herbal blend yielded a significant net reduction in BMI of $0.5,0.9$, and $1.6 \mathrm{~kg} / \mathrm{m}^{2}$ $(P<.0001)$ at 2,4 , and 8 weeks.

\section{Reduction in waist and hip circumferences}

The reduction in waist and hip circumferences, after supplementation with placebo or herbal blend, is summarized
Table 2. Demographic and Baseline Characteristics of the Trial Subjects

\begin{tabular}{lccc}
\hline Characteristics & $\begin{array}{c}\text { Placebo } \\
(\mathrm{n}=46)\end{array}$ & $\begin{array}{c}\text { Herbal blend } \\
(\mathrm{n}=49)\end{array}$ & P value \\
\hline Sex $([\mathrm{n}]$ male $+[n]$ female $)$ & $11 \mathrm{M}+35 \mathrm{~F}$ & $12 \mathrm{M}+37 \mathrm{~F}$ & $\mathrm{NA}$ \\
Age $($ years $)$ & $38.3 \pm 1.2$ & $37.4 \pm 1.0$ & .6 \\
Height $(\mathrm{m})$ & $1.6 \pm 0.01$ & $1.6 \pm 0.01$ & .8 \\
Body weight $(\mathrm{kg})$ & $82.3 \pm 1.5$ & $81.2 \pm 1.2$ & .6 \\
BMI $\left(\mathrm{kg} / \mathrm{m}^{2}\right)$ & $33.8 \pm 0.4$ & $33.6 \pm 0.4$ & .5 \\
Waist circumference $(\mathrm{cm})$ & $97.7 \pm 1.1$ & $98.9 \pm 1.2$ & .6 \\
Hip circumference $(\mathrm{cm})$ & $116.1 \pm 1.1$ & $115.8 \pm 1.0$ & .7 \\
Systolic blood pressure $(\mathrm{mm})$ & $122.4 \pm 2.0$ & $124.5 \pm 2.0$ & .6 \\
Diastolic blood pressure $(\mathrm{mm})$ & $80.7 \pm 1.1$ & $83.7 \pm 1.4$ & .1 \\
Pulse rate (bpm) & $73.4 \pm 0.9$ & $73.8 \pm 1.1$ & .9 \\
\hline
\end{tabular}

Values are expressed as mean $\pm \mathrm{SEM}$.

NA, not applicable.
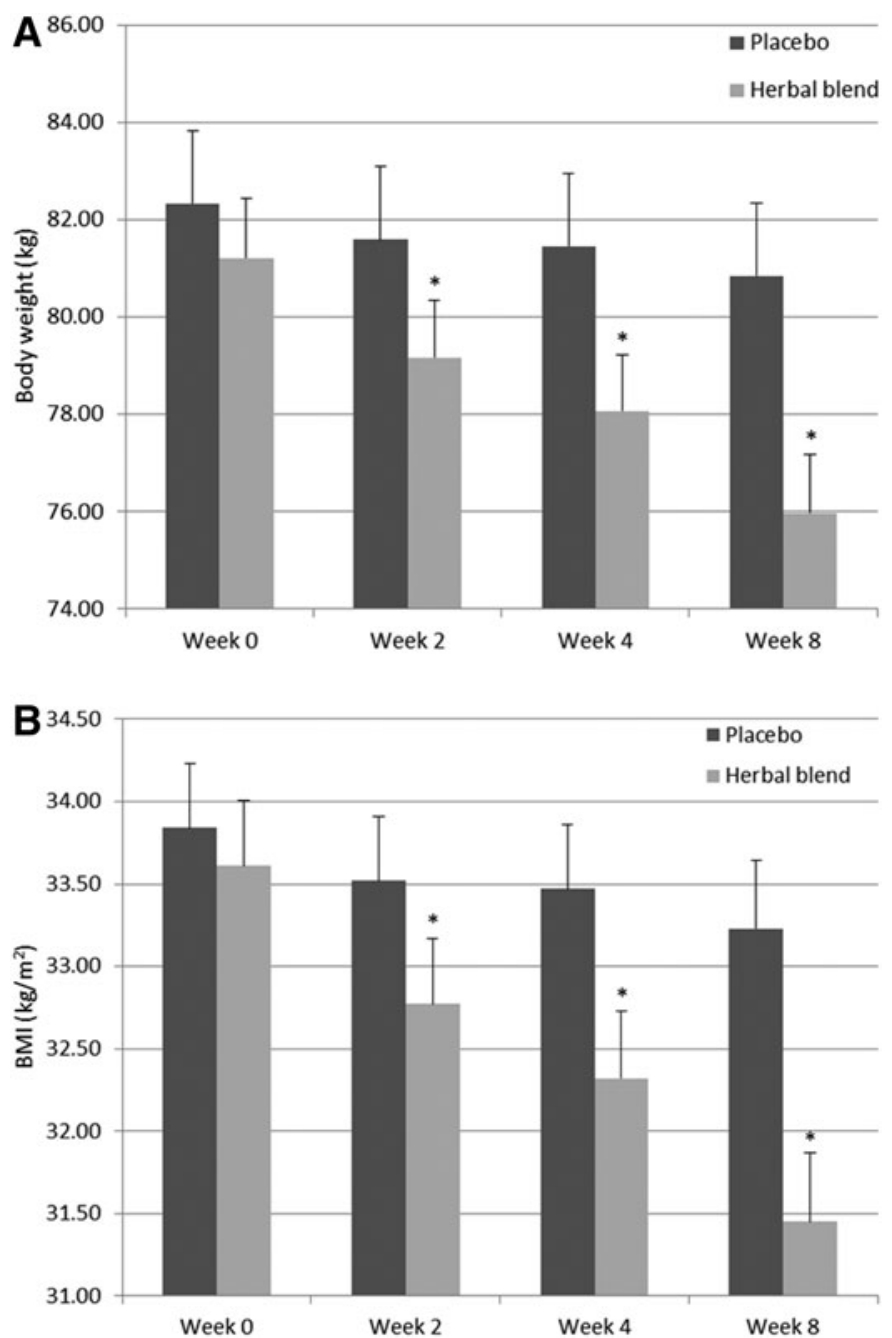

FIG. 3. (A) Body weight and (B) body mass index (BMI) in the herbal blend and placebo groups at 2, 4, and 8 weeks of supplementation. Values represents mean \pm SEM. *Significant difference between the herbal blend $(n=49)$ and placebo $(n=46)$ group, $P<.0001$. 
Table 3. Reduction in Body Weight, Body Mass Index, Waist Circumference, Hip Circumference, and Waist to Hip Ratio in the Herbal Blend and Placebo Groups at 2, 4, and 8 Weeks

\begin{tabular}{|c|c|c|c|c|c|}
\hline Parameters & Weeks & Placebo $(\mathrm{n}=46)$ & Herbal blend $(\mathrm{n}=49)$ & Net reduction ${ }^{\mathrm{a}, \mathrm{b}}$ & $\mathrm{P}$ value \\
\hline \multirow[t]{3}{*}{ Reduction in body weight (kg) } & 2 & $0.8 \pm 0.1$ & $2.0 \pm 0.1$ & $1.3(1.6 \%)$ & $<.0001 *$ \\
\hline & 4 & $0.9 \pm 0.1$ & $3.1 \pm 0.2$ & $2.3(2.8 \%)$ & $<.0001 *$ \\
\hline & 8 & $1.5 \pm 0.2$ & $5.2 \pm 0.3$ & $3.8(4.6 \%)$ & $<.0001^{*}$ \\
\hline \multirow[t]{3}{*}{ Reduction in BMI $\left(\mathrm{kg} / \mathrm{m}^{2}\right)$} & 2 & $0.3 \pm 0.1$ & $0.8 \pm 0.1$ & $0.5(1.5 \%)$ & $<.0001 *$ \\
\hline & 4 & $0.4 \pm 0.1$ & $1.3 \pm 0.1$ & $0.9(2.7 \%)$ & $<.0001 *$ \\
\hline & 8 & $0.6 \pm 0.1$ & $2.2 \pm 0.1$ & $1.6(4.6 \%)$ & $<.0001 *$ \\
\hline \multirow[t]{3}{*}{ Reduction in waist circumference $(\mathrm{cm})$} & 2 & $0.6 \pm 0.6$ & $4.2 \pm 0.5$ & $3.5(3.6 \%)$ & $<.0001 *$ \\
\hline & 4 & $4.0 \pm 0.7$ & $7.7 \pm 0.7$ & $3.7(3.7 \%)$ & $<.0001 *$ \\
\hline & 8 & $6.0 \pm 0.8$ & $11.9 \pm 0.7$ & $5.9(6.0 \%)$ & $<.0001^{*}$ \\
\hline \multirow[t]{3}{*}{ Reduction in hip circumference $(\mathrm{cm})$} & 2 & $0.9 \pm 0.5$ & $2.6 \pm 0.4$ & $1.6(1.4 \%)$ & $.01 *$ \\
\hline & 4 & $1.2 \pm 0.5$ & $3.5 \pm 0.5$ & $2.3(2.0 \%)$ & $.002 *$ \\
\hline & 8 & $3.1 \pm 0.6$ & $6.3 \pm 0.5$ & $3.2(2.7 \%)$ & $.0001 *$ \\
\hline \multirow[t]{3}{*}{ Reduction in waist to hip ratio } & 2 & $0.0 \pm 0.01$ & $0.02 \pm 0.01$ & $0.02(2.3 \%)$ & $.02 *$ \\
\hline & 4 & $0.03 \pm 0.01$ & $0.04 \pm 0.01$ & $0.01(1.2 \%)$ & .1 \\
\hline & 8 & $0.03 \pm 0.01$ & $0.06 \pm 0.01$ & $0.03(3.5 \%)$ & $.00 *$ \\
\hline
\end{tabular}

Values represent mean \pm SEM.

*Significant difference between the herbal blend and placebo group.

${ }^{\mathrm{a}} \mathrm{Net}$ reduction $=$ herbal blend minus placebo.

${ }^{\mathrm{b}}$ Numbers in parentheses represent the \% net reduction from baseline for the herbal group.

in Table 3. Supplementation with the herbal blend resulted in net decreases $(P<.0001)$ in waist and hip circumferences of 5.9 and $3.2 \mathrm{~cm}$, respectively, at the end of the study. This corresponds to a $6.0 \%$ reduction in waist and a $2.7 \%$ reduction in hip size versus baseline. It was also found that the net impact of the herbal blend on waist and hip circumference was statistically significant as early as 2 weeks of supplementation (reduction $=3.5 \mathrm{~cm}, P<.0001$ and $1.6 \mathrm{~cm}$, $P=.01$, respectively). At both 2 and 8 weeks, the reduction in waist to hip ratio (WHR) for the herbal cohort was statistically significant compared with placebo.

\section{Modulation of serum adiponectin levels}

Figure 4 illustrates that herbal supplementation for 8 weeks resulted in a significant increase of $22.5 \%$ in adiponectin levels $(P=.0008)$ compared with placebo. These results suggest that the herbal blend may influence lipid metabolism. ${ }^{16,17}$

\section{Changes in blood lipid profile and fasting glucose}

An analysis of the subject's baseline blood chemistries and anthropomorphic profile indicates that a large proportion of the trial participants in both cohorts had low high-density lipoproteins, high triglycerides, high fasting glucose levels, and large waist size. It is worth noting that herbal supplementation significantly decreased total cholesterol and triglyceride levels by $13.8 \%(P=.002)$ and $41.6 \%(P<.0001)$, respectively (Table 4$)$. In addition, a significant reduction in fasting blood glucose level $(12.2 \%, P<.01)$ was observed in the herbal blend group. Interestingly, the fasting glucose values were within the normal range at the end of 8 -week herbal supplementation $(109.3 \mathrm{mg} / \mathrm{dL}$ vs. $95.9 \mathrm{mg} / \mathrm{dL}, P=.01$; Table 4).

\section{Assessessment of IWQOL}

Figure 5 summarizes changes in mean scores from baseline for four areas of functioning. Results suggest a significant improvement in physical function and self-esteem in the herbal supplemented subjects $(P<.05)$ compared with

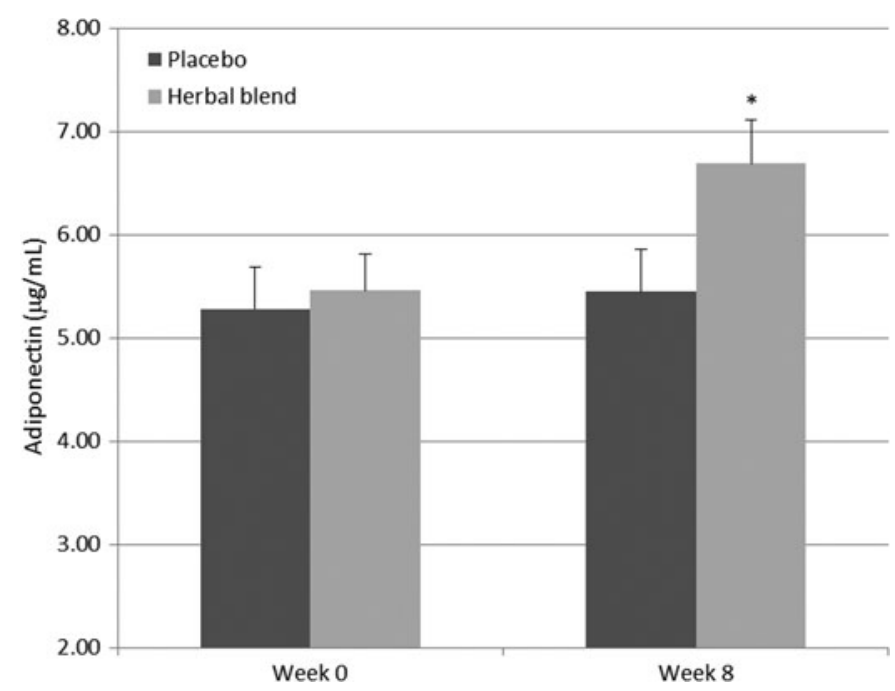

FIG. 4. Serum adiponectin levels in the herbal blend and placebo groups over 8 weeks. Values represents mean \pm SEM. *Significant difference between the herbal blend $(n=45)$ and placebo $(n=42)$ group, $P=.0008$. 
Table 4. Lipid Profile and Glucose Assessment at Baseline and 8 Weeks in Placebo and the Herbal Blend Groups

\begin{tabular}{|c|c|c|c|c|c|c|c|}
\hline \multirow[b]{2}{*}{ Metabolic marker (units) } & \multirow[b]{2}{*}{$\begin{array}{l}\text { Normal } \\
\text { range }\end{array}$} & \multicolumn{3}{|c|}{ Baseline } & \multicolumn{3}{|c|}{ Week 8} \\
\hline & & $\begin{array}{l}\text { Placebo } \\
(\mathrm{n}=46)\end{array}$ & $\begin{array}{l}\text { Herbal blend } \\
\quad(\mathrm{n}=49)\end{array}$ & $\mathrm{P}$ value & $\begin{array}{l}\text { Placebo } \\
(\mathrm{n}=46)\end{array}$ & $\begin{array}{l}\text { Herbal blend } \\
\quad(\mathrm{n}=49)\end{array}$ & $\mathrm{P}$ value \\
\hline Cholesterol (mg/dL) & $<200$ & $198.9 \pm 6.2$ & $204.9 \pm 5.4$ & .4524 & $187.4 \pm 5.1$ & $176.6 \pm 4.4$ & $.0024 *$ \\
\hline Triglycerides (mg/dL) & $10-150$ & $157.9 \pm 7.5$ & $163.7 \pm 7.0$ & .5257 & $117.1 \pm 7.5$ & $95.6 \pm 3.9$ & $<.0001 *$ \\
\hline $\mathrm{LDL}(\mathrm{mg} / \mathrm{dL})$ & $<130$ & $140.6 \pm 1.4$ & $142.6 \pm 1.7$ & .3921 & $139.9 \pm 1.3$ & $136.9 \pm 2.4$ & .1162 \\
\hline HDL (mg/dL) & $>40-60$ & $34.7 \pm 0.8$ & $34.6 \pm 0.8$ & .9073 & $35.6 \pm 0.8$ & $37.2 \pm 0.8$ & .1139 \\
\hline LHR & NA & $4.1 \pm 0.1$ & $4.2 \pm 0.1$ & .5786 & $4.0 \pm 0.1$ & $3.8 \pm 0.1$ & .0606 \\
\hline Fasting glucose (mg/dL) & $<100$ & $107.7 \pm 1.7$ & $109.3 \pm 2.6$ & .7400 & $100.7 \pm 1.3$ & $95.9 \pm 1.5$ & $.0100 *$ \\
\hline
\end{tabular}

Results are presented as mean \pm SEM. Normal ranges were obtained from Medline. ${ }^{14}$

*Significant difference between placebo and the herbal groups.

LDL, low-density lipoproteins; HDL, high-density lipoproteins; LHR, LDL/HDL ratio.

placebo. No significant differences were observed in public distress or work scale between the two groups.

\section{Adverse events}

No major adverse events were reported during this study. Minor adverse events such as headache, nausea, gastrointestinal irritation, plus back, leg, ankle, and joint pain were noted at various evaluation time points. The severity of the adverse events was recorded (Table 5). However, since these events were evenly distributed between the two groups, they are not likely to be associated with herbal supplementation.

\section{Safety assessment}

In order to assess safety, we studied the impact of herbal supplementation on various hematologic parameters as well as other biochemical parameters associated with liver, heart, kidney, and metabolic function. No changes were observed across these organ function panels or in multiple vital signs that included systolic and diastolic blood pressure as well as pulse rate (Table 6). Within-group analysis, comparing

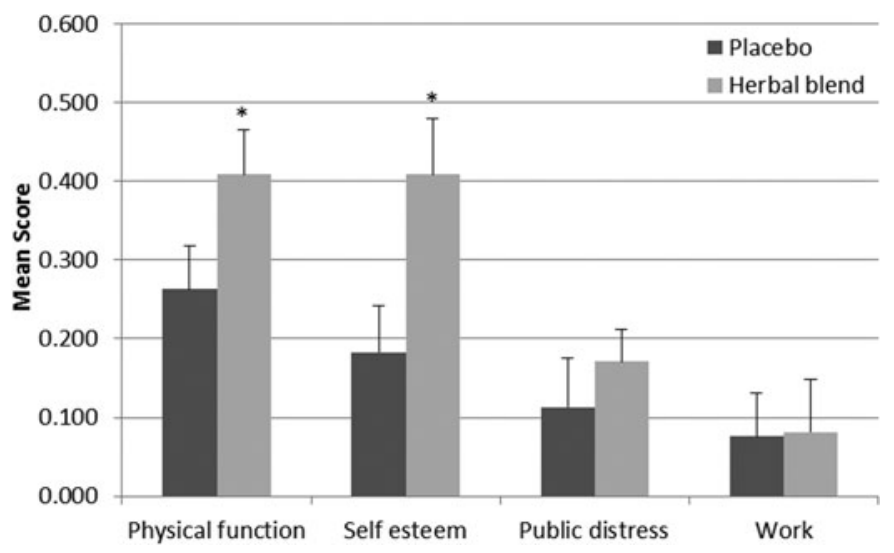

FIG. 5. Summary of mean scores from the impact of weight on quality of life questionnaire in the herbal blend and placebo groups at 8 weeks of supplementation. Values are expressed as mean \pm SEM. *Significant difference between the herbal blend $(n=49)$ and placebo $(n=46)$ group, $P<.05$. values at 8 weeks to baseline, showed a significant decrease in BUN levels for the placebo and herbal groups. These changes were of a similar magnitude for the two groups and may be attributed to the change in diet imposed during the studies, as all subjects were provided with a standardized daily diet of $2000 \mathrm{kcal}$ comprising $61 \%$ carbohydrate, $14 \%$ protein, and $25 \%$ fat. Since the creatinine levels were within the normal range, it suggested that the subjects may be on a high protein diet before participating in the clinical studies.

We also performed electrocardiogram (ECG) evaluations at baseline and subsequent visits to assess whether the herbal supplementation had any adverse effects on cardiac function. The evaluation of the ECGs revealed no significant changes in any of the ECG parameters. Minor ECG variations, which included sinus tachycardia, sinus bradycardia, and primary heart block, were noted in 14 instances among different subjects. All the sporadic changes recorded were within normal limits and were distributed equally among the herbal supplementation and placebo groups. A more important observation was the finding that four of the seven ECG variations in the supplemented group occurred at baseline; whereas only one of the seven ECG variations in the placebo group occurred at this time point (Table 7). Two subjects in the placebo group presented with repeat ECG variations (sinus bradycardia and primary heart block). One subject in the supplemented cohort presented with a repeat ECG variation (sinus bradycardia) that was first noted at baseline. Since these heart function variations were either not consistently seen at all study visits or were first noted at baseline, and were not associated with any abnormalities in blood pressure or pulse rate in all instances, it was inferred

Table 5. Summary of Analysis of Adverse Events IN All SubJects

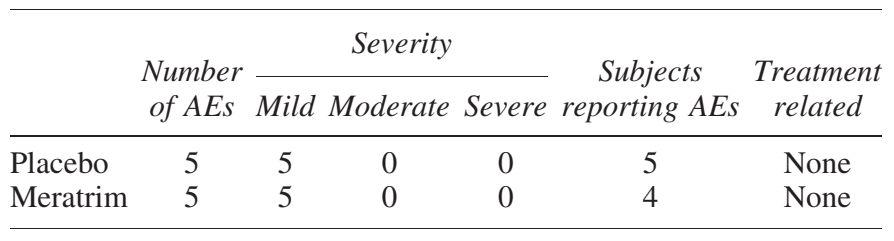

Common adverse events (AEs) reported were headache, nausea, gastrointestinal irritation, general weakness, back, leg, ankle, and joint pain. 
Table 6. Safety Parameter Assessment at Baseline and 8 Weeks in Placebo and the Herbal Groups

\begin{tabular}{|c|c|c|c|c|c|c|c|}
\hline \multirow[b]{2}{*}{ Parameter (units) } & \multirow[b]{2}{*}{$\begin{array}{l}\text { Normal } \\
\text { range }\end{array}$} & \multicolumn{3}{|c|}{ Baseline } & \multicolumn{3}{|c|}{ Week 8} \\
\hline & & $\begin{array}{c}\text { Placebo } \\
(\mathrm{n}=46)\end{array}$ & $\begin{array}{l}\text { Herbal blend } \\
\qquad(\mathrm{n}=49)\end{array}$ & $\mathrm{P}$ value & $\begin{array}{c}\text { Placebo } \\
(\mathrm{n}=46)\end{array}$ & $\begin{array}{l}\text { Herbal blend } \\
\qquad(\mathrm{n}=49)\end{array}$ & $\mathrm{P}$ value \\
\hline \multicolumn{8}{|l|}{ Hematology } \\
\hline Hemoglobin (mg/dL) & $\begin{array}{c}\text { M: } 13.8-17.2 \\
\text { F: } 12.1-15.1\end{array}$ & $12.1 \pm 0.2$ & $11.8 \pm 0.2$ & .3879 & $11.9 \pm 0.2$ & $11.8 \pm 0.2$ & .7 \\
\hline MCH (pg) & $27-31$ & $26.1 \pm 0.5$ & $25.9 \pm 0.5$ & .6228 & $26.0 \pm 0.4$ & $25.4 \pm 0.5$ & .2 \\
\hline $\mathrm{MCHC}(\%)$ & $32-36$ & $30.5 \pm 0.2$ & $30.6 \pm 0.2$ & .9597 & $32.8 \pm 0.5$ & $32.6 \pm 0.5$ & .7 \\
\hline MCV (fL) & $80-100$ & $85.2 \pm 1.0$ & $84.1 \pm 1.1$ & .4272 & $79.3 \pm 1.2$ & $78.1 \pm 1.2$ & .4 \\
\hline $\mathrm{PCV}(\%)$ & $\begin{array}{c}\text { M: } 40.7-50.3 \\
\text { F: } 36.1-44.3\end{array}$ & $39.4 \pm 0.6$ & $38.6 \pm 0.6$ & .3296 & $36.5 \pm 0.7$ & $36.2 \pm 0.7$ & .0 \\
\hline RBC (million/mm³) & $4.7-6.1$ & $4.6 \pm 0.1$ & $4.6 \pm 0.1$ & .6604 & $4.6 \pm 0.1$ & $4.7 \pm 0.1$ & .4 \\
\hline $\mathrm{WBC}\left(\times 1000 / \mathrm{mm}^{3}\right)$ & $4.5-10$ & $8.1 \pm 0.2$ & $7.9 \pm 0.3$ & .5137 & $7.1 \pm 0.3$ & $6.9 \pm 0.3$ & .6 \\
\hline Platelet count $\left(\times 1000 / \mathrm{mm}^{3}\right)$ & $150-450$ & $325.8 \pm 11.8$ & $331.1 \pm 11.7$ & .9599 & $351.6 \pm 11.2$ & $360.1 \pm 12.8$ & .6 \\
\hline \multicolumn{8}{|l|}{ Liver function } \\
\hline Total protein $(\mathrm{mg} / \mathrm{dL})$ & $6-8.3$ & $7.2 \pm 0.1$ & $7.1 \pm 0.1$ & .4251 & $7.3 \pm 0.1$ & $7.3 \pm 0.1$ & .8 \\
\hline ALP (IU/L) & $44-147$ & $147.7 \pm 4.6$ & $138.5 \pm 3.8$ & .0584 & $131.2 \pm 3.0$ & $125.1 \pm 2.1$ & .5 \\
\hline SGOT (U/L) & $10-34$ & $25.8 \pm 0.7$ & $26.7 \pm 0.7$ & .6129 & $23.7 \pm 0.7$ & $23.5 \pm 0.6$ & .8 \\
\hline SGPT (U/L) & $5-35$ & $33.5 \pm 1.1$ & $33.3 \pm 1.0$ & .6901 & $31.6 \pm 0.9$ & $30.7 \pm 0.9$ & .5 \\
\hline Total bilirubin (mg/dL) & $0.3-1.9$ & $0.7 \pm 0.03$ & $0.7 \pm 0.03$ & .8975 & $0.8 \pm 0.02$ & $0.7 \pm 0.0$ & .06 \\
\hline \multicolumn{8}{|l|}{ Cardiac function } \\
\hline $\mathrm{CK}(\mathrm{U} / \mathrm{L})$ & $60-400$ & $120.1 \pm 3.0$ & $119.9 \pm 3.0$ & .8616 & $113.6 \pm 1.8$ & $114.5 \pm 2.1$ & .9 \\
\hline Systolic BP (mm Hg) & $<120$ & $122.4 \pm 2.0$ & $124.5 \pm 2.0$ & .5802 & $122.6 \pm 1.6$ & $121.8 \pm 1.6$ & .2 \\
\hline Diastolic BP (mm Hg) & $<80$ & $80.7 \pm 1.1$ & $83.7 \pm 1.4$ & .1376 & $80.8 \pm 1.3$ & $80.1 \pm 1.1$ & .08 \\
\hline Pulse rate (beats/min) & $60-100$ & $73.4 \pm 0.9$ & $73.8 \pm 1.1$ & .9449 & $70.7 \pm 0.8$ & $72.8 \pm 1.0$ & .1 \\
\hline \multicolumn{8}{|l|}{ Renal function } \\
\hline Creatinine $(\mathrm{mg} / \mathrm{dL})$ & $\begin{array}{c}\text { M: } 0.7-1.3 \\
\text { F: } 0.6-1.1\end{array}$ & $0.9 \pm 0.01$ & $0.8 \pm 0.01$ & .1733 & $0.9 \pm 0.01$ & $0.9 \pm 0.01$ & .5 \\
\hline BUN (mg/dL) & $\begin{array}{c}\text { M: } 8-24 \\
\text { F: 6-21 }\end{array}$ & $33.3 \pm 1.0$ & $34.0 \pm 1.0$ & .7128 & $25.7 \pm 0.5$ & $26.4 \pm 0.6$ & .3 \\
\hline
\end{tabular}

Results are presented as mean \pm SEM. Normal ranges were obtained from Medline and the Mayo Clinic. ${ }^{14,15}$

$\mathrm{MCH}$, mean corpuscular hemoglobin volume; MCHC, mean corpuscular hemoglobin concentration; MCV, mean corpuscular volume; PCV, packed cell volume;

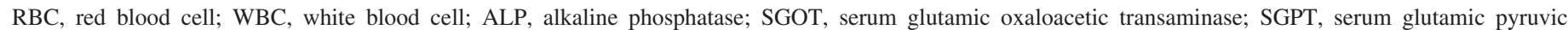
transaminase; CK, creatine kinase; BP, blood pressure; M, Male; F, Female.

that these ECG variations were not linked with herbal supplementation.

Overall, the absence of any consistent supplement-related abnormalities in the herbal group suggests that it has no adverse effect on heart function.

\section{Dropouts}

Five subjects (one from the herbal group and four from the placebo group) were dropped from the study and were lost to follow-up (Fig. 2). No subject discontinued partici- pating in the study as a result of an adverse event. The results attributed to the dropouts were excluded from all statistical analyses as per the predefined criteria established in the study protocol.

\section{DISCUSSION}

The objective of the current analysis was to evaluate the efficacy and tolerability of an herbal blend in promoting healthy weight loss. We combined the data from two randomized, double-blind, placebo-controlled, clinical trials

Table 7. Electrocardiogram Assessment in the Herbal Blend and Placebo Groups

\begin{tabular}{|c|c|c|c|c|}
\hline \multirow[b]{2}{*}{ Study groups } & \multicolumn{4}{|c|}{ Electrocardiogram Findings } \\
\hline & Day 0 & Week 2 & Week 4 & Week 8 \\
\hline Placebo & $\begin{array}{l}\text { Normal }(n=45) \\
\text { Sinus tachycardia }(n=1)\end{array}$ & $\begin{array}{l}\text { Normal }(n=43) \\
\text { Sinus bradycardia }(n=2) \\
\text { Primary heart block }(n=1)\end{array}$ & $\begin{array}{l}\text { Normal }(n=44) \\
\text { Sinus bradycardia }(n=2)\end{array}$ & $\begin{array}{l}\text { Normal }(n=45) \\
\text { Primary heart block }(n=1)\end{array}$ \\
\hline Herbal blend & $\begin{array}{l}\text { Normal }(n=45) \\
\text { Sinus tachycardia }(n=3) \\
\text { Sinus bradycardia }(n=1)\end{array}$ & $\begin{array}{l}\text { Normal }(n=48) \\
\text { Sinus bradycardia }(n=1)\end{array}$ & Normal $(n=49)$ & $\begin{array}{l}\text { Normal }(n=47) \\
\text { Sinus bradycardia }(n=1) \\
\text { Primary heart block }(n=1)\end{array}$ \\
\hline
\end{tabular}


conducted in 100 human subjects with a BMI between 30 and $40 \mathrm{~kg} / \mathrm{m}^{2}$ in order to achieve higher statistical power, thereby providing a broader insight into the beneficial effects of the herbal blend in managing weight.

The main findings of these two studies corroborate previous results that the herbal blend, in combination with a standard diet $(2000 \mathrm{kcal} / \mathrm{day})$ and moderate daily exercise, produces a clinically significant incremental reduction in body weight, waist, and hip circumference compared with diet management and exercise alone. As reported earlier, this additional benefit was an average weight loss of $3.8 \mathrm{~kg}$ beyond the average weight loss of $1.5 \mathrm{~kg}$ for the control group. We also observed that the incremental weight loss elicited by the herbal blend were statistically significant by 2 weeks of supplementation, and it included statistically significant reductions in waist and hip circumferences. Although we did not measure changes in body composition, the significant reduction in WHR observed in these studies $(P<.001)$ suggests a reduction and possible redistribution of body fat, as high WHR values are known surrogate indicators of visceral fat levels. ${ }^{18-21}$ This is an important finding, as it suggests that the herbal blend may be more effective in reducing visceral over subcutaneous fat tissue, thereby allowing for possible body reshaping and improvements in metabolic parameters. These relatively fast beneficial effects, plus the availability of free food, may have encouraged the participants to remain on the trial program, potentially reducing the dropout rate. ${ }^{22}$

Previous unpublished in vitro data and results from these clinical studies suggest that the herbal ingredient mediates its weight loss effect via multiple mechanisms. Addition of the herbal ingredient to cultures of 3T3-L1 adipocytes resulted in a marked reduction in peroxisome proliferatoractivated receptor gamma (PPAR $\gamma)$, cluster of differentiation 36 (CD36), and adipocyte-differentiation related protein (ADRP) levels, and reduced the expression of perilipin protein on the surface of lipid droplets. These results suggest, respectively, that the herbal blend may attenuate fat accumulation by partially blocking adipogenesis and fat uptake, and by rendering lipid droplets more susceptible to lipases. ${ }^{23-28}$ The ability of the herbal mix to promote significant weight loss may explain the higher levels of circulating adiponectin observed in the herbal supplemented group. These higher adiponectin levels, in turn, may increase fat metabolism, leading to a reduction in WHR, as adiponectin has been shown to facilitate fat oxidation in skeletal muscle and liver. ${ }^{29,30}$

The subjects selected for the current trials presented with mild dyslipidemia and elevated fasting glucose (Table 4). This was expected, as obese subjects who are otherwise healthy generally exhibit this clinical phenotype. ${ }^{31,32}$ However, at study conclusion, both cholesterol and triglyceride levels were significantly improved toward the normal range in the supplement cohort but not in placebo. Similarly, fasting glucose levels were reduced to $95 \mathrm{mg} / \mathrm{dL}$ in the treated group, whereas placebo glucose levels remained above $100 \mathrm{mg} / \mathrm{dL}$. These changes may result from the significant increase in adiponectin levels that occurred in the supplement cohort coupled with their higher overall weight loss. Circulating adiponectin concentrations have been shown to inversely associate with levels of adiposity and fasting glucose levels but correlate positively with insulin sensitivity in obese, nonobese, and nondiabetic populations. ${ }^{33}$ Therefore, it appears that the herbal blend, when combined with lifestyle changes, may modulate several key pathways that are involved with the metabolism of fat and glucose.

It is well established that obesity adversely affects physical health, emotional well-being, and psychosocial functioning. ${ }^{13,34-36}$ Consistent with these findings, quality of life parameters improved significantly more for subjects consuming the herbal supplement. Obese subjects who underwent bariatric surgery also demonstrated marked improvement in health-related quality of life. ${ }^{37}$

The herbal blend was well tolerated in both clinical trials. No major adverse events were reported from the use of the herbal blend. Only a few minor adverse events were reported in some participants, which were evenly distributed between the placebo and the herbal group. There was no change in blood biomarkers related to liver, kidney, and heart function, nor did we note any changes in subject vital signs. Further, we did not observe any abnormalities in ECG profile due to the herbal supplementation.

In summary, supplementation with the herbal blend at a daily dose of $800 \mathrm{mg}$ resulted in statistically significant reductions in body weight, BMI, and waist and hip circumference that exceed those achieved via diet and exercise alone. The blend's significant effect on body weight and anthropomorphic parameters occurred as early as 2 weeks. Supplementation with the herbal blend also resulted in a significant improvement in blood lipid profile and glycemic control. Assessment of various safety parameters related to liver, kidney, and heart function, plus the lack of significant adverse events during the eight-week studies indicate that the herbal blend is well tolerated. Since the studies described here are of a relatively short duration, longer-term trials are needed to ascertain the herbal blend's efficacy and safety over an extended period of use. Results from the current studies suggest that the herbal blend appears to be an effective and well-tolerated ingredient for weight management.

\section{ACKNOWLEDGMENT}

This work was supported by an unrestricted grant from InterHealth Nutraceuticals Inc., Benicia, CA, to J.S.S.

\section{AUTHOR DISCLOSURE STATEMENT}

No competing financial interests exist.

\section{REFERENCES}

1. WHO: Obesity and Overweight. Fact sheet No. 311. www.who .int/mediacentre/factsheets/fs311/en/index.html (accessed November 2012). 
2. Faust IM, Johnson PR, Stern JS, et al: : Diet-induced adipocyte number increase in adult rats: a new model of obesity. Am J Physiol 1978;235:E279-E286.

3. Klyde BJ, Hirsch J: Increased cellular proliferation in adipose tissue of adult rats fed a high-fat diet. J Lipid Res 1979;20: 705-715.

4. Klyde BJ, Hirsch J: Isotopic labeling of DNA in rat adipose tissue: evidence for proliferating cells associated with mature adipocytes. J Lipid Res 1979;20:691-704.

5. Mallare JT, Karabell AH, Velasquez-Mieyer P, et al.: Current and future treatment of metabolic syndrome and type 2 diabetes in children and adolescents. Diabetes Spectrum 2005;18:220-228.

6. Schnee DM, Zaiken K, McCloskey WW: An update on the pharmacological treatment of obesity. Curr Med Res Opin 2006; 22:1463-1474.

7. Bray GA, Ryan DH: Drug treatment of the overweight patient. Gastroenterology 2007;132:2239-2252.

8. Kang JG, Park CY: Anti-obesity drugs: a review about their effects and safety. Diabetes Metab J 2012;36:13-25.

9. Hubbard VS, Hall WH: Gastrointestinal Surgery for Severe Obesity. Obes Surg 1991;1:257-265.

10. Pittler MH, Ernst E: Dietary supplements for body-weight reduction: a systematic review. Am J Clin Nutr 2004;79:529-536.

11. Pillitteri JL, Shiffman S, Rohay JM, et al.: Use of dietary supplements for weight loss in the United States: results of a national survey. Obesity (Silver Spring) 2008;16:790-796.

12. Kolotkin RL, Head S, Hamilton M, et al.: Assessing impact of weight on quality of life. Obes Res 1995;3:49-56.

13. Kolotkin RL, Crosby RD, Kosloski KD, et al.: Development of a brief measure to assess quality of life in obesity. Obes Res 2001; 9:102-111.

14. ADAM, Inc.: Medical encyclopedia: MedlinePlus. www.nlm .nih.gov/medlineplus.gov/medlineplus/encyclopedia.html (accessed November 2012).

15. Mayo Foundation for Medical Education and Research: Mayo Clinic. www.mayoclinic.com (accessed November 2012).

16. Guebre-Egziabher F, Bernhard J, Funahashi T, et al:: Adiponectin in chronic kidney disease is related more to metabolic disturbances than to decline in renal function. Nephrol Dial Transplant 2005;20:129-134.

17. Kern PA, Di Gregorio GB, Lu T, et al.: Adiponectin expression from human adipose tissue: relation to obesity, insulin resistance, and tumor necrosis factor-alpha expression. Diabetes 2003;52: 1779-1785.

18. Elsayed EF, Tighiouart H, Weiner DE, et al.: Waist-to-hip ratio and body mass index as risk factors for cardiovascular events in CKD. Am J Kidney Dis 2008;52:49-57.

19. Taylor RW, Keil D, Gold EJ, et al.: Body mass index, waist girth, and waist-to-hip ratio as indexes of total and regional adiposity in women: evaluation using receiver operating characteristic curves. Am J Clin Nutr 1998;67:44-49.

20. Janssen I, Katzmarzyk PT, Ross R: Waist circumference and not body mass index explains obesity-related health risk. Am J Clin Nutr 2004;79:379-384.

21. Fox CS, Massaro JM, Hoffmann U, et al.: Abdominal visceral and subcutaneous adipose tissue compartments: association with metabolic risk factors in the Framingham Heart Study. Circulation 2007;116:39-48.

22. Stern JS, Hirsch J, Blair SN, et al.: Weighing the options: criteria for evaluating weight-management programs. The Committee to Develop Criteria for Evaluating the Outcomes of Approaches to Prevent and Treat Obesity. Obes Res 1995;3: 591-604.

23. Brun RP, Spiegelman BM: PPAR gamma and the molecular control of adipogenesis. J Endocrinol 1997;155:217-218.

24. Yamauchi T, Waki H, Kamon J, et al.: Inhibition of RXR and PPARgamma ameliorates diet-induced obesity and type 2 diabetes. J Clin Invest 2001;108:1001-1013.

25. Jones JR, Barrick C, Kim KA, et al:: Deletion of PPARgamma in adipose tissues of mice protects against high fat diet-induced obesity and insulin resistance. Proc Natl Acad Sci U S A 2005; 102:6207-6212.

26. Bonen A, Tandon NN, Glatz JF, et al.: The fatty acid transporter FAT/CD36 is upregulated in subcutaneous and visceral adipose tissues in human obesity and type 2 diabetes. Int J Obes (Lond) 2006;30:877-883.

27. Faleck DM, Ali K, Roat R, et al.: Adipose differentiation-related protein regulates lipids and insulin in pancreatic islets. Am J Physiol Endocrinol Metab 2010;299:E249-E257.

28. Tansey JT, Sztalryd C, Gruia-Gray J, et al.: Perilipin ablation results in a lean mouse with aberrant adipocyte lipolysis, enhanced leptin production, and resistance to diet-induced obesity. Proc Natl Acad Sci U S A 2001;98:6494-6499.

29. Yamauchi T, Kamon J, Minokoshi Y, et al.: Adiponectin stimulates glucose utilization and fatty-acid oxidation by activating AMP-activated protein kinase. Nat Med 2002;8:12881295.

30. Yamauchi T, Kamon J, Waki H, et al.: The fat-derived hormone adiponectin reverses insulin resistance associated with both lipoatrophy and obesity. Nat Med 2001;7:941-946.

31. Wang H, Peng DQ: New insights into the mechanism of low high-density lipoprotein cholesterol in obesity. Lipids Health Dis 2011;10:176.

32. Martyn JA, Kaneki M, Yasuhara S: Obesity-induced insulin resistance and hyperglycemia: etiologic factors and molecular mechanisms. Anesthesiology 2008;109:137-148.

33. Stefan N, Vozarova B, Funahashi T, et al:: Plasma adiponectin concentration is associated with skeletal muscle insulin receptor tyrosine phosphorylation, and low plasma concentration precedes a decrease in whole-body insulin sensitivity in humans. Diabetes 2002;51:1884-1888.

34. Riazi A, Shakoor S, Dundas I, et al.: Health-related quality of life in a clinical sample of obese children and adolescents. Health Qual Life Outcomes 2010;8:134.

35. Swallen KC, Reither EN, Haas SA, et al.: Overweight, obesity, and health-related quality of life among adolescents: the National Longitudinal Study of Adolescent Health. Pediatrics 2005;115: 340-347.

36. Kawachi I: Physical and psychological consequences of weight gain. J Clin Psychiatry 1999;60 Suppl 21:5-9.

37. Loux TJ, Haricharan RN, Clements RH, et al.: Health-related quality of life before and after bariatric surgery in adolescents. J Pediatr Surg 2008;43:1275-1279. 